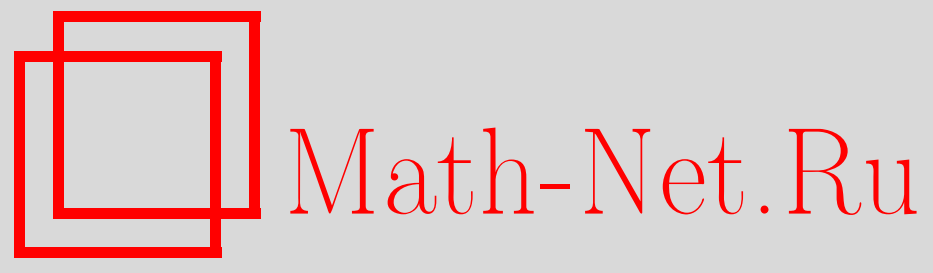

А. В. Карпишков, М. А. Нефёдов, В. А. Салеев, А. В. Шипилова, Рождение $D$-мезонов на Большом адронном коллайдере в реджевском пределе КХД, Вестн. Сам. гос. техн. ун-та. Сер. Физ.-мат. науки, 2015, номер 3, 441-461

DOI: https://doi.org/10.14498/vsgtu1402

Использование Общероссийского математического портала MathNet.Ru подразумевает, что вы прочитали и согласны с пользовательским соглашением

http://www.mathnet.ru/rus/agreement

Параметры загрузки:

IP : 54.224 .135 .184

26 апреля 2023 г., 04:57:34

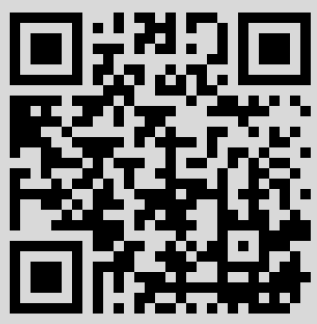


Вестн. Сам. гос. техн. ун-та. Сер. Физ.-мат. науки. 2015. Т. 19, № 3. С. $441-461$

ISSN: 2310-7081 (online), 1991-8615 (print)

doi: http://dx.doi.org/10.14498/vsgtu1402

УДК 517.958:539.1; 539.126.4

\title{
РОЖДЕНИЕ $D$-МЕЗОНОВ НА БОЛЬШОМ АДРОННОМ КОЛЛАЙДЕРЕ В РЕДЖЕВСКОМ ПРЕДЕЛЕ КХД*
}

\author{
А. В. Карпишков, М. А. Нефёдов, \\ В. А. Салеев, А. В. Шипилова \\ Самарский государственный университет, \\ Россия, 443011, Самара, ул. Академика Павлова, 1.
}

\section{Аннотация}

В работе рассматривается инклюзивное рождение очарованных $D^{0}, D^{+}$, $D^{\star+}$ и $D_{s}^{+}$мезонов в протон-антипротонных соударениях на коллайдере Тэватрон и протон-протонных соударениях на Большом адронном коллайдере (БАК). Теоретические расчёты проведены в лидирующем порядке теории реджезованных партонов (ТРП), основанной на эффективной квантовой теории поля Л. Н. Липатова и подходе $k_{T^{-}}$ факторизации при высоких энергиях. В работе были использованы универсальные функции фрагментации $(\Phi \Phi)$, полученные фитированием данных рождения $D$-мезонов на линейном электрон-позитронном коллайдере LEP1. Нами описаны распределения $D$-мезонов по поперечному импульсу, измеренные в центральной области быстрот коллаборациями CDF на Тэватроне $(|y|<1)$ и ALICE на БАК $(|y|<0.5)$ в пределах ошибок измерений и без привлечения свободных параметров. На стадии численных расчётов нами использовались неинтегрированные глюонные функции распределения (ФР) Кимбера, Мартина и Рыскина (KMP).

Ключевые слова: квантовая хромодинамика, $k_{T}$-факторизация, теория реджезованных партонов, физика высоких энергий, очарованные мезоны.

doi: http://dx.doi.org/10.14498/vsgtu1402

(C) 2015 Самарский государственный технический университет.

\section{Образец для цитирования}

К арпиишов А. В., Нефёдов М. А., Салеев В. А., Шипилова А. В. Рождение $D$ мезонов на Большом адронном коллайдере в реджевском пределе КХД // Becmн. Cам. гос. техн. ун-та. Сер. Физ.-мат. науки, 2015. Т. 19, № 3. С. 441-461. doi: 10.14498/vsgtu1402.

\section{Сведения об авторах}

Антон Витальевич Карпишков (karpishkov@rambler.ru), аспирант, каф. общей и теоретической физики.

Максим Александрович Нефёдов (nefedovma@gmail.com), аспирант, каф. общей и теоретической физики.

Владимир Анатольевич Салеев (д.ф.-м.н., проф.; saleev@samsu.ru; автор, ведущий переписку), профессор, каф. общей и теоретической физики.

Александра Викторовна Шипилова (к.ф.-м.н.; alexshipilova@samsu.ru), научный сотрудник, лаб. математического моделирования материалов Межвузовского Центра теоретического материаловедения СамГУ.

*Настоящая статья представляет собой расширенный вариант доклада [1], сделанного авторами на Четвёртой международной конференции «Математическая физика и её приложения» (Россия, Самара, 25 августа - 1 сентября 2014). 
Введение. Изучение рождения очарованных мезонов с большими поперечными импульсами в высокоэнергетических адронных столкновениях предоставляет возможность для проверки пертурбативной квантовой хромодинамики (KХД). В процессах рождения таких мезонов $\mu \geqslant m$, где $\mu$ - характерный энергетический масштаб сильного взаимодействия, имеющий порядок поперечного импульса рождённой частицы, а $m$ - масса тяжёлого кварка, что обеспечивает малость константы сильного взаимодействия $\alpha_{s}(\mu) \ll 1$. Эти процессы также несут информацию о новом динамическом режиме пертурбативной КХД, называемом пределом Редже, который характеризуется условием $\sqrt{S} \gg \mu \gg \Lambda$, где $\sqrt{S}$ - инвариантная энергия столкновения в системе центра масс сталкивающихся адронов, а $\Lambda$ - асимптотический масштабный параметр КХД. В пределе Редже появляется новый малый параметр $x \sim \mu / \sqrt{S}$, который с увеличением энергии становится всё более малым, что приводит к появлению вклада логарифмов нового типа $\left(\alpha_{s} \log (1 / x)\right)^{n}$ во всех порядках теории возмущений по константе сильного взаимодействия.

Существуют различные пертурбативные поправки, доминирующие в разных процессах и областях фазового пространства. Так, в случае рождения тяжёлых конечных состояний (бозонов Хиггса, пар кварков $(t \bar{t})$, парных струй с большими инвариантными массами или Дрелл-Яновских пар) начальные партоны имеют относительно большую долю импульса $x \sim 0.1$ и поправки в основном обусловлены излучением мягких коллинеарных глюонов, которые могут увеличивать сечение на фактор 2. Напротив, относительно лёгкие конечные состояния (тяжёлые кварконии с малыми поперечными импульсами, одиночные струи, прямые фотоны или парные струи с малыми инвариантными массами) рождаются путём слияния партонов с малыми значениями $x$, характерное значение $x \sim 10^{-3}$ для энергий $\sqrt{S} \sim 1$ ТэВ. Радиационные поправки к этим процессам обусловлены рождением дополнительных жёстких струй. Единственный способ описания подобных процессов в традиционной коллинеарной партонной модели (KПМ) - это вычисление поправок высших порядков по степеням константы сильного взаимодействия $\alpha_{s}=g_{s}^{2} / 4 \pi \mathrm{k}$ амплитудам рассеяния, что представляет собой сложную задачу для некоторых процессов уже в следующем за лидирующим порядке (СЛП). Чтобы преодолеть эти трудности и учесть значительную часть поправок высших порядков в режиме малых $x$, был предложен подход $k_{T}$-факторизации, или факторизации при высоких энергиях [2-4].

Недавно коллаборация ALICE измерила дифференциальные сечения $d \sigma / d p_{T}$ для инклюзивного рождения $D^{0}, D^{+}, D^{\star+}$ и $D_{s}^{+}$мезонов $[5,6]$ в протон-протонных столкновениях на ускорителе БАК в ЦЕРНе $(\sqrt{S}=7$ ТэВ $)$ как функцию поперечного момента $\left(p_{T}\right)$-мезонов в центральной области быстрот, $|y|<0.5$. Эти измерения дополняют данные коллаборации CDF [7], полученные ранее в протон-антипротонных столкновениях на ускорителе Тэватрон в области быстрот $|y|<1.0$ и при энергии столкновения $\sqrt{S}=1.96$ ТэВ.

Спектры $D$-мезонов изучались в СЛП КПМ в рамках двух подходов: в так называемых схемах GM-VFN [8-12] и FONNL [13]. В первой схеме, реализованной в работах [14-16], фрагментационные логарифмы, доминирующие при $p_{T} \gg m$, пересуммированы путём введения функций фрагментации, удовлетворяющих эволюционным уравнениям Докшицера-ГрибоваЛипатова-Альтарелли-Паризи (ДГЛАП) [17-19]. При этом сохранена за- 
висимость сечения жёсткого рассеяния от массы очарованного кварка для правильного описания спектров $D$-мезонов в области $p_{T} \sim m$. ФФ партонов в $D$-мезоны были получены как в лидирующем, так и в следующим за ним порядках в схеме GM-VFN с помощью фитирования данных, полученных в $e^{+} e^{-}$аннигиляции. Для этого использовались данные коллаборации OPAL с ускорителя LEP1 в ЦЕРНе [20,21]. Напротив, в подходе FONNL поправки к лидирующему порядку в сечение рождения $D$-мезонов вычислены с использованием непертурбативных $\Phi \Phi ~ c$-кварков в $D$-мезон, не являющихся результатом ДГЛАП [17-19] эволюции. Схема FONNL была развита в работах $[22,23]$ и заключается в вычислении квадратов модулей матричных элементов партонных подпроцессов в СЛП и суммировании логарифмов с большими поперечными импульсами в главном логарифмическом приближении для процессов рождения тяжёлых кварков. Для обеспечения самосогласованности вычислений в FONNL схеме $[22,23]$ процесс получения непертурбативных ФФ и схема вычислений сечений тяжёлых кварков связаны между собой и должны использоваться вместе. Спектры $D$-мезонов, измеренные коллаборацией CDF [7] на Тэватроне, а также коллаборацией ALICE [5, 6] на БАК, достаточно хорошо описываются в подходах GM-VFNS [14-16] и FONNL [22,23] в рамках экспериментальных погрешностей.

Целью настоящей работы является исследование рождения $D$-мезонов на Тэватроне и БАК в рамках подхода факторизации при высоких энергиях, называемого также подходом $k_{T}$-факторизации [2-4], дополненной калибровочно-инвариантными амплитудами с реджезованными глюонами в начальном состоянии [42-45]. Далее такую комбинацию мы будем называть теорией реджезованных партонов (ТРП).

Исследование фрагментационного рождения $D$-мезонов в $k_{T}$-факторизации было проведено в недавней работе [24] с использованием виртуальных глюонов в начальном состоянии и формализма партонных $\Phi Р$, зависящих от поперечного импульса. В работе [24] показано, что учёт рождения $D$-мезонов только через процесс глюонного слияния в $c \bar{c}$-пару недостаточен для описания экспериментальных данных коллаборации ALICE [5]. Согласно расчётам в работе [24], этот результат может свидетельствовать о необходимости учёта вклада многопартонного рассеяния в рождение $D$-мезонов.

Мы считаем ТРП теоретически более последовательной, так как она основана на калибровочно-инвариантной эффективной теории процессов в квазимультиреджевской кинематике (KMPK) КХД, а также сохраняет калибровочную инвариантность амплитуд и может быть обобщена на вычисления в СЛП. Недавно ТРП была успешно применена для анализа инклюзивного рождения одиночных струй [25], парных струй [26], прямых фотонов [27, 28], фотона и струи [29], лептонных пар Дрелла-Яна [30], b-кварковых струй $[31,32]$, рождения чармония и боттомония [33-38] на Тэватроне и БАК. Данные исследования продемонстрировали преимущества схемы факторизации при высоких энергиях, основанной на подходе реджезованных партонов, при описании экспериментальных данных в сравнении с вычислениями в КПМ.

1. Модель. Феноменология сильных взаимодействий при высоких энергиях указывает на доминирующую роль процесса слияния глюонов в кваркантикварковую пару при рождении тяжёлых мезонов. Как показано в работе [15], значительный вклад в сечение рождения $D$-мезонов вносит фраг- 
ментация глюонов и $c$-кварков в $D$-мезон, в то время как вклад фрагментации лёгких кварков пренебрежимо мал. Исходя из этого в нашей работе мы рассмотрим только вклады фрагментации $c$-кварков и глюонов в рождение различных $D$-мезонов.

В адронных столкновениях сечения жёстких процессов могут быть представлены в виде свёртки зависящих от масштаба партонных (кварковых или глюонных) распределений и квадрата модуля амплитуды жёсткого партонного рассеяния. Указанные распределения соответствуют плотностям партонов в протоне с продольной долей импульса $x$, проинтегрированным по всем значениям поперечного импульса вплоть до $k_{T}=\mu$. Их эволюция от масштаба $\mu_{0}$, который связан с непертурбативным режимом, до характерного масштаба $\mu$ описывается уравнениями эволюции ДГЛАП [17-19], которые позволяют просуммировать большие логарифмы вида $\log \left(\mu^{2} / \Lambda_{Q C D}^{2}\right)$ (коллинеарные логарифмы). Характерный масштаб $\mu$ процессов жёсткого рассеяния обычно имеет порядок поперечной массы $m_{T}=\sqrt{m^{2}+\left|\mathbf{p}_{T}\right|^{2}}$ рождающейся частицы (или адронной струи) с (инвариантной) массой $m$ и поперечным импульсом $\mathbf{p}_{T}$. С увеличением энергии, когда величина $x \sim \mu / \sqrt{S}$ становится малой, всё более весомый вклад начинает вносить новый тип логарифмов $\log (1 / x)$. Их вклад может становиться даже большим, чем вклад коллинеарных логарифмов. Такие логарифмы входят как в партонные распределения, так и в партонные сечения и могут быть пересуммированы в подходе БалицкогоФадина-Кураева-Липатова (БФКЛ) [39-41]. Этот подход описывает КХД амплитуды рассеяний в области больших $S$ и фиксированного переданного импульса $t$, при $S \gg|t|$ (область Редже), с различными цветовыми состояниями в $t$-канале.

Реджезация глюонов имеет место в условиях специальной кинематики процессов при высоких энергиях. При больших $\sqrt{S}$ доминирующие вклады в сечения КХД процессов даёт мультиреджевская кинематика (MPK), в которой все частицы имеют ограниченные (не растущие с $\sqrt{S}$ ) поперечные импульсы или объединены в струи с ограниченной инвариантной массой каждой струи и большими (растущими с $\sqrt{S}$ ) инвариантными массами любой пары струй. В порядке логарифмического приближения, следующем за лидирующим, струя может содержать пары партонов (два глюона или кваркантикварковую пару). Такая кинематика называется квазимультиреджевской кинематикой (KMPK). Несмотря на большое количество диаграмм Фейнмана, дающих ненулевые вклады, оказывается, что в борновском приближении MPK амплитуды приобретают простую факторизованную форму. Более того, радиационные поправки к таким амплитудам не искажают эту форму, а их зависимость от энергии обусловлена факторами Редже $s_{i}^{\omega\left(q_{i}\right)}$, где $s_{i}-$ инвариантные массы пар соседних струй, а $\omega\left(q_{i}\right)$ можно интерпретировать как отклонение параметра траектории Редже глюона от единицы, зависящее от переданного импульса. Такое явление называется реджезацией глюона.

Важная роль отведена вершинам взаимодействий реджезованных глюонов с обычными глюонами и кварками. Для их определения можно использовать один из двух способов: «классический» БФКЛ метод, основанный на свойствах аналитичности и унитарности амплитуд рождения частиц в условиях реджевской кинематики [42], или они могут быть получены непосред- 
ственно из неабелевого калибровочно инвариантного эффективного действия для взаимодействий реджезованных партонов с обычными партонами в КХД. Этот метод впервые был предложен в работе [43] для реджезованных глюонов, а в работе [44] - для реджезованных кварков. Полный набор правила Фейнмана для индуцированных и эффективных вершин можно найти в работах $[44,45]$.

Новым объектом в подходе БФКЛ являются неинтегрированные по поперечному импульсу партонные $\Phi$ Р $\Phi\left(x, t, \mu^{2}\right)$, зависящие от виртуальности партона $t=-\left|\mathbf{q}_{T}\right|^{2}$, где $\mathbf{q}_{T}-$ его поперечный импульс. Неинтегрированные $\Phi P$ связаны с соответствующими коллинеарными $Ф Р$ через условие нормировки:

$$
x G\left(x, \mu^{2}\right)=\int^{\mu^{2}} d t \Phi\left(x, t, \mu^{2}\right) .
$$

В ЛП ТРП $D$-мезоны рождаются путём фрагментации глюонов, рождённых в процессах $2 \rightarrow 1$, или $c$-кварков из процессов $2 \rightarrow 2$ :

$$
\begin{aligned}
& \mathscr{R}+\mathscr{R} \rightarrow g, \\
& \mathscr{R}+\mathscr{R} \rightarrow c+\bar{c},
\end{aligned}
$$

где $\mathscr{R}$ - реджезованный глюон. В соответствии с правилами, описанными в работе [45], амплитуды рассматриваемых процессов (1) и (2) могут быть получены из диаграмм Фейнмана, изображённых на рис. 1 и 2, где штриховой линией обозначены реджезованные глюоны.

Определим 4-векторы

$$
\left(n^{+}\right)^{\mu}=P_{2}^{\mu} / E_{2}, \quad\left(n^{-}\right)^{\mu}=P_{1}^{\mu} / E_{1},
$$

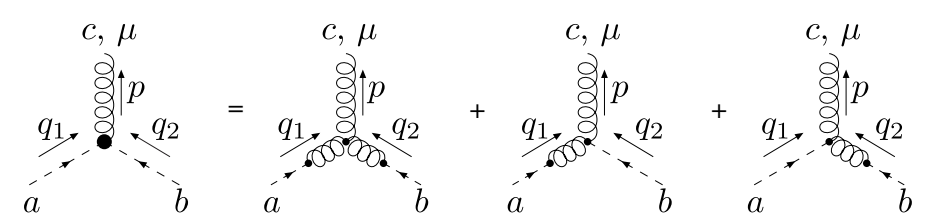

Рис. 1. Диаграммы Фейнмана для подпроцессов (1)

[Figure 1. Feynman diagrams for the subprocess (1)]
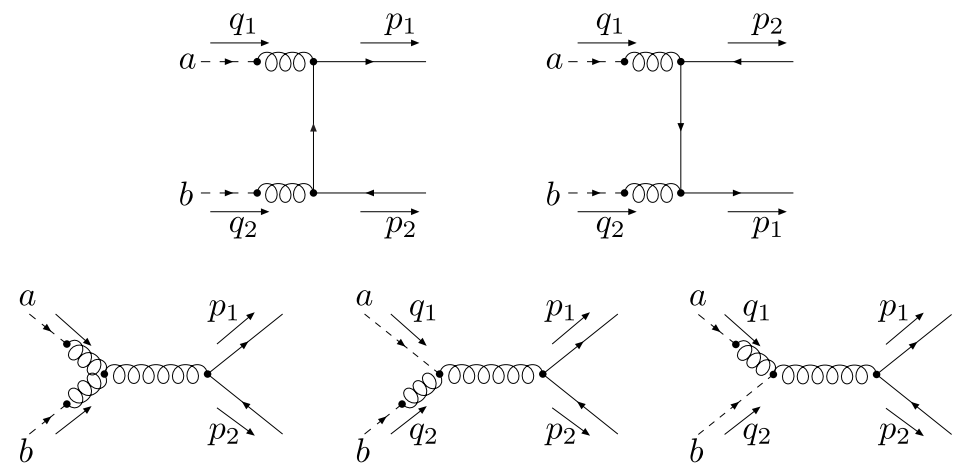

Рис. 2. Диаграммы Фейнмана для подпроцессов (2)

[Figure 2 Feynman diagrams for the subprocess (2)] 
где $P_{1,2}^{\mu}-4$-импульсы сталкивающихся протонов, а $E_{1,2}-$ их энергии. Тогда выполняются следующие соотношения:

$$
\left(n^{ \pm}\right)^{2}=0, \quad n^{+} \cdot n^{-}=2, \quad S=\left(P_{1}+P_{2}\right)^{2}=4 E_{1} E_{2}
$$

Для произвольного 4-импульса $k^{\mu}$ имеем $k^{ \pm}=k \cdot n^{ \pm}$. Четырёхмерные импульсы реджезованных глюонов могут быть представлены в виде

$$
q_{1}^{\mu}=\frac{q_{1}^{+}}{2}\left(n^{-}\right)^{\mu}+q_{1 T}^{\mu}, \quad q_{2}^{\mu}=\frac{q_{2}^{-}}{2}\left(n^{+}\right)^{\mu}+q_{2 T}^{\mu},
$$

где $q_{T}=\left(0, \mathbf{q}_{T}, 0\right)$. Амплитуда рождения глюона через столкновение двух реджезованных глюонов может быть представлена в виде скалярного произведения эффективной вершины Фадина-Кураева-Липатова $C_{\mathscr{R} \mathscr{R}}^{g, \mu}\left(q_{1}, q_{2}\right)$ и 4-вектора поляризации конечного глюона $\varepsilon_{\mu}(p)$ :

$$
\mathscr{M}(\mathscr{R}+\mathscr{R} \rightarrow g)=C_{\mathscr{R} \mathscr{R}}^{g, \mu}\left(q_{1}, q_{2}\right) \varepsilon_{\mu}(p)
$$

где

$$
\begin{aligned}
C_{\mathscr{R} \mathscr{R}}^{g, \mu}\left(q_{1}, q_{2}\right)= & -\sqrt{4 \pi \alpha_{s}} f^{a b c} \frac{q_{1}^{+} q_{2}^{-}}{2 \sqrt{t_{1} t_{2}}} \times \\
& \times\left[\left(q_{1}-q_{2}\right)^{\mu}+\frac{\left(n^{+}\right)^{\mu}}{q_{1}^{+}}\left(q_{2}^{2}+q_{1}^{+} q_{2}^{-}\right)-\frac{\left(n^{-}\right)^{\mu}}{q_{2}^{-}}\left(q_{1}^{2}+q_{1}^{+} q_{2}^{-}\right)\right],
\end{aligned}
$$

$a$ и $b$ - цветовые индексы реджезованных глюонов со входящими 4-импульсами $q_{1}$ и $q_{2}, f^{a b c}\left(a=1, \ldots, N_{c}^{2}-1\right)$ - антисимметричная структурная константа цветовой калибровочной группы $S U_{C}(3)$. Квадрат амплитуды партонного подпроцесса $\mathscr{R}+\mathscr{R} \rightarrow g$ можно получить непосредственно из $(3)$ :

$$
\overline{|\mathscr{M}(\mathscr{R}+\mathscr{R} \rightarrow g)|^{2}}=\frac{3}{2} \pi \alpha_{s} \mathbf{p}_{T}^{2}
$$

Амплитуда процесса (2) может быть представлена в виде суммы трёх амплитуд

$$
\mathscr{M}(\mathscr{R}+\mathscr{R} \rightarrow c+\bar{c})=\mathscr{M}_{1}+\mathscr{M}_{2}+\mathscr{M}_{3}
$$

где

$$
\begin{aligned}
& \mathscr{M}_{1}=-i \pi \alpha_{s} \frac{q_{1}^{+} q_{2}^{-}}{\sqrt{t_{1} t_{2}}} T^{a} T^{b} \bar{U}\left(p_{1}\right) \gamma^{\alpha} \frac{\hat{p}_{1}-\hat{q}_{1}}{\left(p_{1}-q_{1}\right)^{2}} \gamma^{\beta} V\left(p_{2}\right)\left(n^{+}\right)^{\alpha}\left(n^{-}\right)^{\beta}, \\
& \mathscr{M}_{2}=-i \pi \alpha_{s} \frac{q_{1}^{+} q_{2}^{-}}{\sqrt{t_{1} t_{2}}} T^{b} T^{a} \bar{U}\left(p_{1}\right) \gamma^{\beta} \frac{\hat{p}_{1}-\hat{q}_{2}}{\left(p_{1}-q_{2}\right)^{2}} \gamma^{\alpha} V\left(p_{2}\right)\left(n^{+}\right)^{\alpha}\left(n^{-}\right)^{\beta}, \\
& \mathscr{M}_{3}= 2 \pi \alpha_{s} \frac{q_{1}^{+} q_{2}^{-}}{\sqrt{t_{1} t_{2}}} T^{c} f^{a b c} \frac{\bar{U}\left(p_{1}\right) \gamma^{\mu} V\left(p_{2}\right)}{\left(p_{1}+p_{2}\right)^{2}} \times \\
& \times\left[\left(n^{-}\right)^{\mu}\left(q_{2}^{+}+\frac{q_{2}^{2}}{q_{1}^{-}}\right)-\left(n^{+}\right)^{\mu}\left(q_{1}^{-}+\frac{q_{1}^{2}}{q_{2}^{+}}\right)+\left(q_{1}-q_{2}\right)^{\mu}\right],
\end{aligned}
$$


$T^{a}$ - генераторы фундаментального представления цветовой калибровочной группы $S U_{C}(3)$.

Квадрат модуля амплитуды в случае безмассовых кварков записывается в следующем виде:

$$
\overline{|\mathscr{M}(\mathscr{R}+\mathscr{R} \rightarrow c+\bar{c})|^{2}}=16 \pi^{2} \alpha_{s}^{2} \frac{\left(x_{1} x_{2} S\right)^{2}}{t_{1} t_{2}}\left(\frac{1}{2 N_{c}} \mathscr{A}_{\mathrm{Ab}}+\frac{N_{c}}{2\left(N_{c}^{2}-1\right)} \mathscr{A}_{\mathrm{NAb}}\right),
$$

где

$$
\begin{gathered}
\mathscr{A}_{\mathrm{Ab}}=\frac{t_{1} t_{2}}{\hat{t} \hat{u}}-\left(1+\frac{p_{2}^{+}}{\hat{u}}\left(q_{1}^{-}-p_{2}^{-}\right)+\frac{p_{2}^{-}}{\hat{t}}\left(q_{2}^{+}-p_{2}^{+}\right)\right)^{2}, \\
\mathscr{A}_{\mathrm{NAb}}=\frac{2}{S^{2}}\left(\frac{p_{2}^{+}\left(q_{1}^{-}-p_{2}^{-}\right) S}{\hat{u}}+\frac{S}{2}+\frac{\Delta}{\hat{s}}\right)\left(\frac{p_{2}^{-}\left(q_{2}^{+}-p_{2}^{+}\right) S}{\hat{t}}+\frac{S}{2}-\frac{\Delta}{\hat{s}}\right)- \\
-\frac{t_{1} t_{2}}{q_{1}^{-} q_{2}^{+} \hat{s}}\left(\left(\frac{1}{\hat{t}}-\frac{1}{\hat{u}}\right)\left(q_{1}^{-} p_{2}^{+}-q_{2}^{+} p_{2}^{-}\right)+\frac{q_{1}^{-} q_{2}^{+} \hat{s}}{\hat{t} \hat{u}}-2\right), \\
\Delta=\frac{S}{2}\left(\hat{u}-\hat{t}+2 q_{1}^{-} p_{2}^{+}-2 q_{2}^{+} p_{2}^{-}+t_{1} \frac{q_{2}^{+}-2 p_{2}^{+}}{q_{2}^{+}}-t_{2} \frac{q_{1}^{-}-2 p_{2}^{-}}{q_{1}^{-}}\right) .
\end{gathered}
$$

В (4) и (5) верхняя черта указывает на усреднение (суммирование) по всем начальным (конечным) спиновым и цветовым состояниям,

$$
t_{1}=-q_{1}^{2}=\left|\mathbf{q}_{1 T}\right|^{2}, \quad t_{2}=-q_{2}^{2}=\left|\mathbf{q}_{2 T}\right|^{2},
$$

а также

$\hat{s}=\left(q_{1}+q_{2}\right)^{2}=\left(p_{1}+p_{2}\right)^{2}, \quad \hat{t}=\left(q_{1}-p_{1}\right)^{2}=\left(q_{2}-p_{2}\right)^{2}, \quad \hat{u}=\left(q_{2}-p_{1}\right)^{2}=\left(q_{1}-p_{2}\right)^{2}$.

Квадрат модуля амплитуды (5) совпадает с результатом, ранее полученным в работе [2-4]. Мы проверили, что в коллинеарном пределе $q_{(1,2) T}=0$, квадрат модуля амплитуды (5) после усреднения по азимутальным углам переходит в квадрат модуля амплитуды соответствующего партонного подпроцесса в коллинеарной модели, а именно $g+g \rightarrow c+\bar{c}$. Мы проводим наш анализ в области $\sqrt{S}, p_{T} \gg m_{c}$, что оправдывает использование схемы ZM-VFNS, где массами очарованных кварков в амплитуде жёсткого процесса можно пренебречь.

В подходе $k_{T}$-факторизации дифференциальное сечение рассеяния подпроцесса (1) имеет вид

$$
\begin{aligned}
\frac{d \sigma}{d y d p_{T}}(p+p & \rightarrow g+X)= \\
& =\frac{1}{p_{T}^{3}} \int d \phi_{1} \int d t_{1} \Phi\left(x_{1}, t_{1}, \mu^{2}\right) \Phi\left(x_{2}, t_{2}, \mu^{2}\right) \overline{|\mathscr{M}(\mathscr{R}+\mathscr{R} \rightarrow g)|^{2}},
\end{aligned}
$$

где $\phi_{1}$ - азимутальный угол между $\mathbf{p}_{T}$ и $\mathbf{q}_{1 T}$.

Аналогичная формула для подпроцесса (2) может быть записана в виде 


$$
\begin{aligned}
\frac{d \sigma}{d y_{1} d y_{2} d p_{1 T} d p_{2 T}}(p+p & \left.\rightarrow c\left(p_{1}\right)+\bar{c}\left(p_{2}\right)+X\right)= \\
= & \frac{p_{1 T} p_{2 T}}{16 \pi^{3}} \int d \phi_{1} \int d \Delta \phi \int d t_{1} \times \\
& \times \Phi\left(x_{1}, t_{1}, \mu^{2}\right) \Phi\left(x_{2}, t_{2}, \mu^{2}\right) \frac{\overline{|\mathscr{M}(\mathscr{R}+\mathscr{R} \rightarrow c+\bar{c})|^{2}}}{\left(x_{1} x_{2} S\right)^{2}},
\end{aligned}
$$

где $x_{1}=q_{1}^{+} / P_{1}^{+}, x_{2}=q_{2}^{-} / P_{2}^{-} ; \Delta \phi$ - азимутальный угол между $\mathbf{p}_{1 T}$ и $\mathbf{p}_{2 T}$; быстроту конечного партона с 4-импульсом $p$ можно представить в виде $y=(1 / 2) \ln \left(p^{+} / p^{-}\right)$. Легко показать, что в пределе $t_{1,2} \rightarrow 0$ мы возвращаемся от (6) и (7) к стандартной формуле факторизации КПМ.

Важной составляющей в нашей модели являются неинтегрированные глюонные ФР, которые мы получаем согласно методу Кимбера, Мартина и Рыскина (KMP) [46]. Эти распределения получены введением вспомогательной функции, зависящей от одного масштаба и удовлетворяющей объединённому БФКЛ/ДГЛАП уравнению эволюции, в котором лидирующие БФКЛ логарифмы $\alpha_{S} \log (1 / x)$ полностью пересуммированы, а также учитываются приближённо лидирующие БФКЛ эффекты главной (кинематической) части. Эта процедура получения неинтегрированных ФР требует меньших вычислительных усилий, чем точное решение уравнений эволюции с двумя масштабами, например, таких, как уравнение Чиафалони-Катани-ФиораниМарчезини [47-49].

Использование формулы $k_{T}$-факторизации и неинтегрированных ФР с продольной кинематической переменной $(x)$ требует реджезации $t$-канальных партонов. В соответствии с $[43,44]$ реджезованные партоны несут только одну большую компоненту 4-импульса, и, следовательно, их виртуальность обусловлена поперечным импульсом. Такая кинематика $t$-канальных партонов соответствует MPK частиц, рождённых в жёстком процессе. В наших предыдущих работах [33-38], посвящённых процессам рождения тяжёлых кваркониев, мы показали, что эти неинтегрированные ФР КМР дают лучшее описание $p_{T}-$ спектров, измеренных на Тэватроне [50-56] и БАК [57-59].

В модели фрагментации образование $D$-мезона глюоном или $c$-кварком описывается функцией фрагментации $(\Phi \Phi) D_{c, g}\left(z, \mu^{2}\right)$. В соответствии с теоремой факторизации фрагментационной модели формула для сечения рождения $D$-мезона имеет вид [60]

$$
\begin{aligned}
\frac{d \sigma(p+p \rightarrow D+X)}{d p_{D T} d y} & = \\
& =\sum_{i} \int_{0}^{1} \frac{d z}{z} D_{i \rightarrow D}\left(z, \mu^{2}\right) \frac{d \sigma\left(p+p \rightarrow i\left(p_{i}=p_{D} / z\right)+X\right)}{d p_{i T} d y_{i}},
\end{aligned}
$$

где $D_{i \rightarrow D}\left(z, \mu^{2}\right)$ - ФФ партона $i$ в $D$-мезон на масштабе $\mu^{2} ; z$-доля продольного импульса фрагментирующей частицы, уносимая $D$-мезоном. В приближении нулевой массы параметр фрагментации $z$ может быть определён как $p_{D}^{\mu}=z p_{i}^{\mu} ; p_{D}$ и $p_{i}-4$-импульсы $D$-мезона и партона соответственно, a $y_{D}=y_{i}$. В наших вычислениях мы использовали ФФ ЛП из работы [14], где непертурбативные $Ф \Phi$ партонов в $D^{0}, D^{+}, D^{\star+}$ и $D_{s}^{+}$мезоны получены 
на основе данных коллаборации OPAL на коллайдере LEP1 [20,21]. Эти ФФ универсальны и нарушение $\mu$-скейлинга в них подчиняется уравнениям эволюции ДГЛАП. В работах [14-16] параметризации на начальном масштабе $\mu_{0}=m_{c}$ для $Ф \Phi$ взяты в следующем виде:

$$
D_{c}\left(z, \mu_{0}^{2}\right)=N_{c} \frac{z(1-z)^{2}}{\left[(1-z)+\epsilon_{c}\right]^{2}}, \quad D_{g, q}\left(z, \mu_{0}^{2}\right)=0 \text {. }
$$

Поскольку вклад фрагментации глюона при $\mu>\mu_{0}$ обусловлен пертурбативным переходом глюонов в $c \bar{c}$-пару, подчиняющимся уравнениям ДГЛАП эволюции, то часть $c$-кварков, рождённых в подпроцессе (2), с их последующим переходом в $D$-мезон уже учтена в рождении $D$-мезона путём фрагментации глюона. В этом случае, чтобы избежать двойного счёта, мы должны вычесть этот вклад. Это может быть эффективно осуществлено введением обрезания по $\hat{s}$ снизу, то есть по порогу рождения $c \bar{c}$-пары в формуле $(7)$, а именно $\hat{s}>4 m_{c}^{2}$.

2. Результаты расчётов. Первое измерение спектров $D$-мезонов по поперечному импульсу в адрон-адронных столкновениях было выполнено коллаборацией CDF на коллайдере Тэватрон [7] при энергии $\sqrt{S}=1.96$ ТэВ. $D^{0}, D^{+}, D^{\star+}$ и $D_{s}^{+}$мезоны детектировались в центральной области по быстроте $|y|<1.0$ и имели поперечные импульсы вплоть до 20 ГэВ. На рис. 3 показаны данные для $d \sigma / d p_{T}$, в которых усреднён вклад частиц и античастиц в сравнении с нашими предсказаниями в подходе реджезованных партонов. Штриховые линии отвечают вкладу процесса (1), в то время как штрихпунктирные линии соответствуют вкладу процесса (2). Сумма обоих вкладов показана в виде сплошной линии. Мы оценили теоретическую погрешность, возникающую из-за произвола в определении масштабов факторизации и перенормировки, путём варьирования масштабов в пределах $\mu / 2$ и $2 \mu$ около среднего значения $\mu=p_{T}$. Теоретические неопределённости показаны на рисунках заштрихованными областями. Мы обнаружили хорошее согласие между нашими предсказаниями и экспериментальными данными в широком интервале по поперечным импульсам $D$-мезонов в рамках экспериментальных и теоретических погрешностей. Однако наши предсказания хуже описывают экспериментальные данные при малых $p_{T}$, что может быть сигналом о значимости эффектов конечной массы $c$-кварков в области, где $p_{T} \approx m$. В нашем подходе увеличение энергии столкновения при сохранении других кинематических условий должно привести к лучшему согласию между теорией и экспериментом, поскольку мы ожидаем усиления логарифмических вкладов вида $\log \left(p_{T} / \sqrt{S}\right)$ над другими эффектами.

Наши ожидания действительно подтверждаются при описании данных, полученных на БАК при энергии $\sqrt{S}=7$ ТэВ коллаборацией ALICE [5]. Предыдущие предсказания, сделанные в СЛП КПМ, находятся в согласии с данными ALICE, однако мы можем видеть, что FONNL схема [23] имеет тенденцию к превышению относительно данных, а схема GM-VFNS [16] - к занижению. На рис. 4 мы сравниваем наши предсказания с данными ALICE [5], сохраняя обозначения кривых такими же, как на рис. 3. Энергии столкновений на БАК в 2-3.5 раза больше, чем на Тэватроне, а интервал быстрот $D$-мезонов более узкий $(|y|<0.5)$, поэтому на БАК характерное значение $x$ 

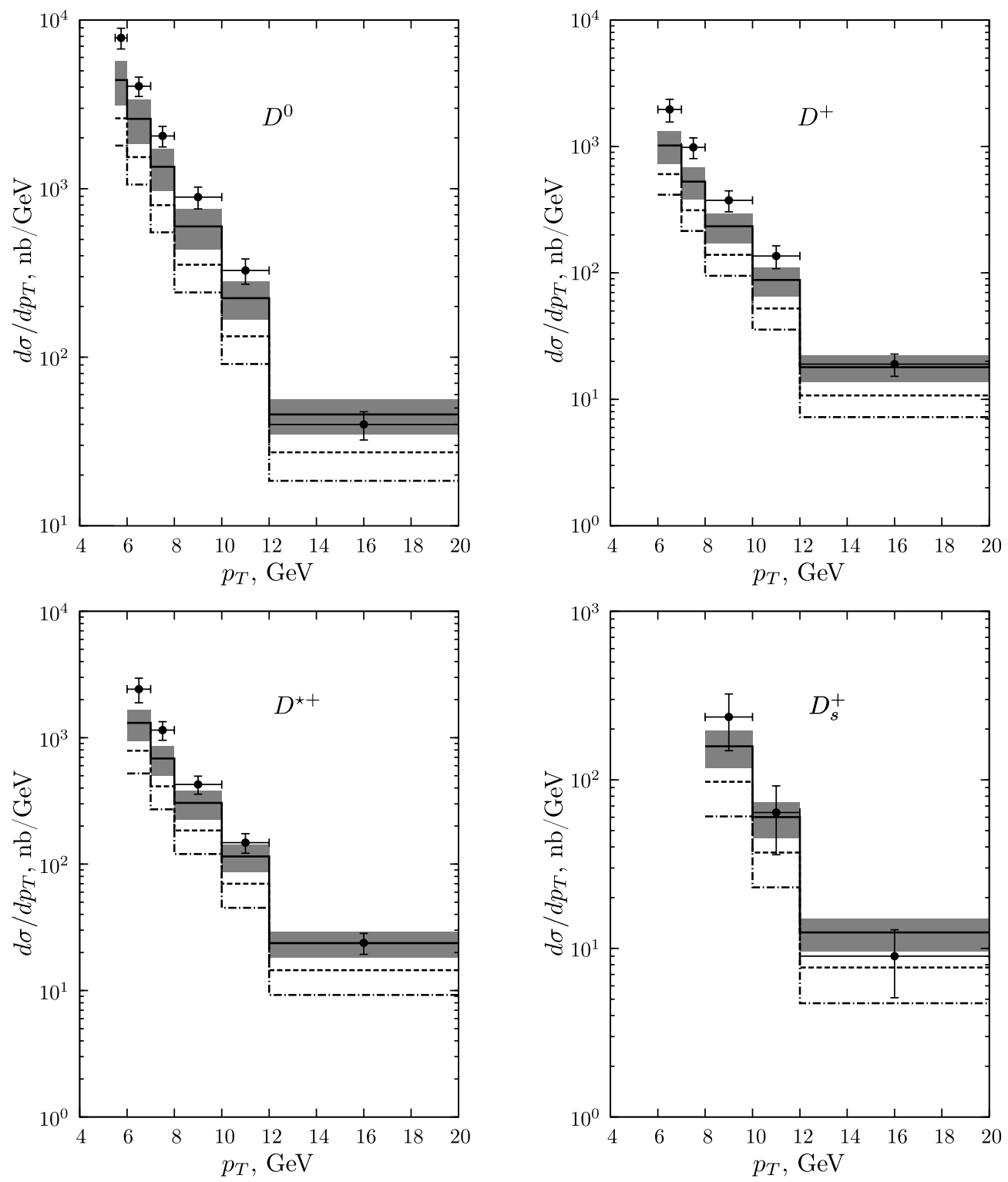

Рис. 3. Распределения по поперечному импульсу $D^{0}$ (вверху слева), $D^{+}$(вверху справа), $D^{\star+}$ (внизу слева), и $D_{s}^{+}$(внизу справа) мезонов в $p \bar{p}$ рассеянии при $\sqrt{S}=1.96$ ТэВ и $|y|<1.0$. Штриховая линия обозначает вклад глюонной фрагментации, штрих-пунктирная - вклад $c$-кварковой фрагментации, сплошная - их сумма. Данные коллаборации CDF Тэватрона взяты из [7]

[Figure 3. Transverse momentum distributions of $D^{0}$ (left, top), $D^{+}$(right, top), $D^{\star+}$ (left, bottom), and $D_{s}^{+}$(right, bottom) mesons in $p \bar{p}$ scattering with $\sqrt{S}=1.96 \mathrm{TeV}$ and $|y|<1.0$.

The dashed line represents the contribution of gluon fragmentation, the dash-dotted line is the $c$-quark-fragmentation contribution, and the black solid line is their sum obtained with KMR UPDF. The CDF data at Tevatron are from Ref. [7]] 

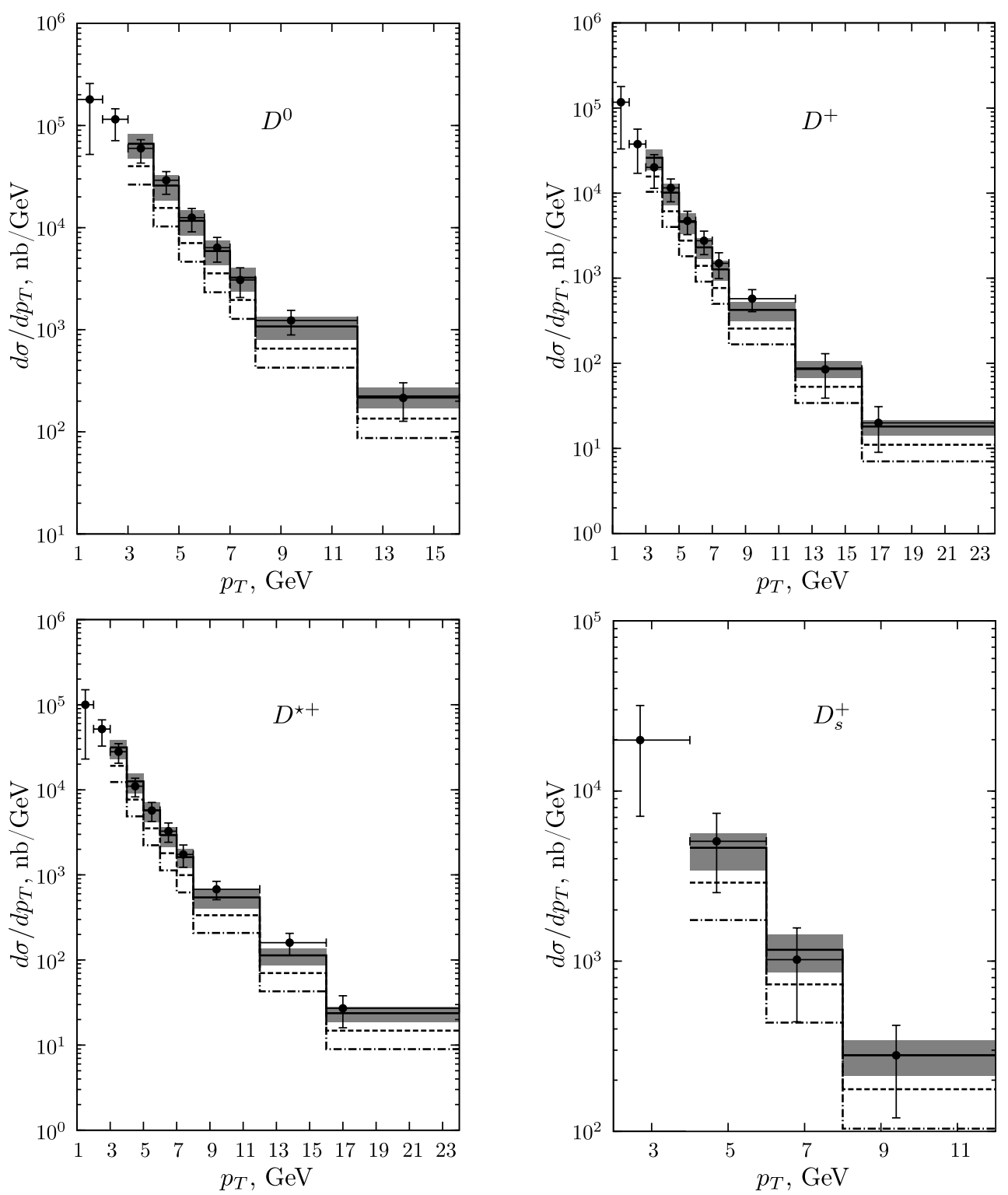

Рис. 4. Распределения по поперечному импульсу $D^{0}$ (вверху слева), $D^{+}$(вверху справа), $D^{\star+}$ (внизу слева), и $D_{s}^{+}$(внизу справа) мезонов в $p p$ рассеянии при $\sqrt{S}=7$ ТэВ и $|y|<0.5$.

Обозначения те же, что и на рис. 3. Данные коллаборации ALICE БAK взяты из [5]

[Figure 4. Transverse momentum distributions of $D^{0}$ (left, top), $D^{+}$(right, top), $D^{\star+}$ (left, bottom), and $D_{s}^{+}$(right, bottom) mesons in $p p$ scattering with $\sqrt{S}=7 \mathrm{TeV}$ and $|y|<0.5$.

The notations are as in Fig. 3. The ALICE data at LHC are from Ref. [5]] 

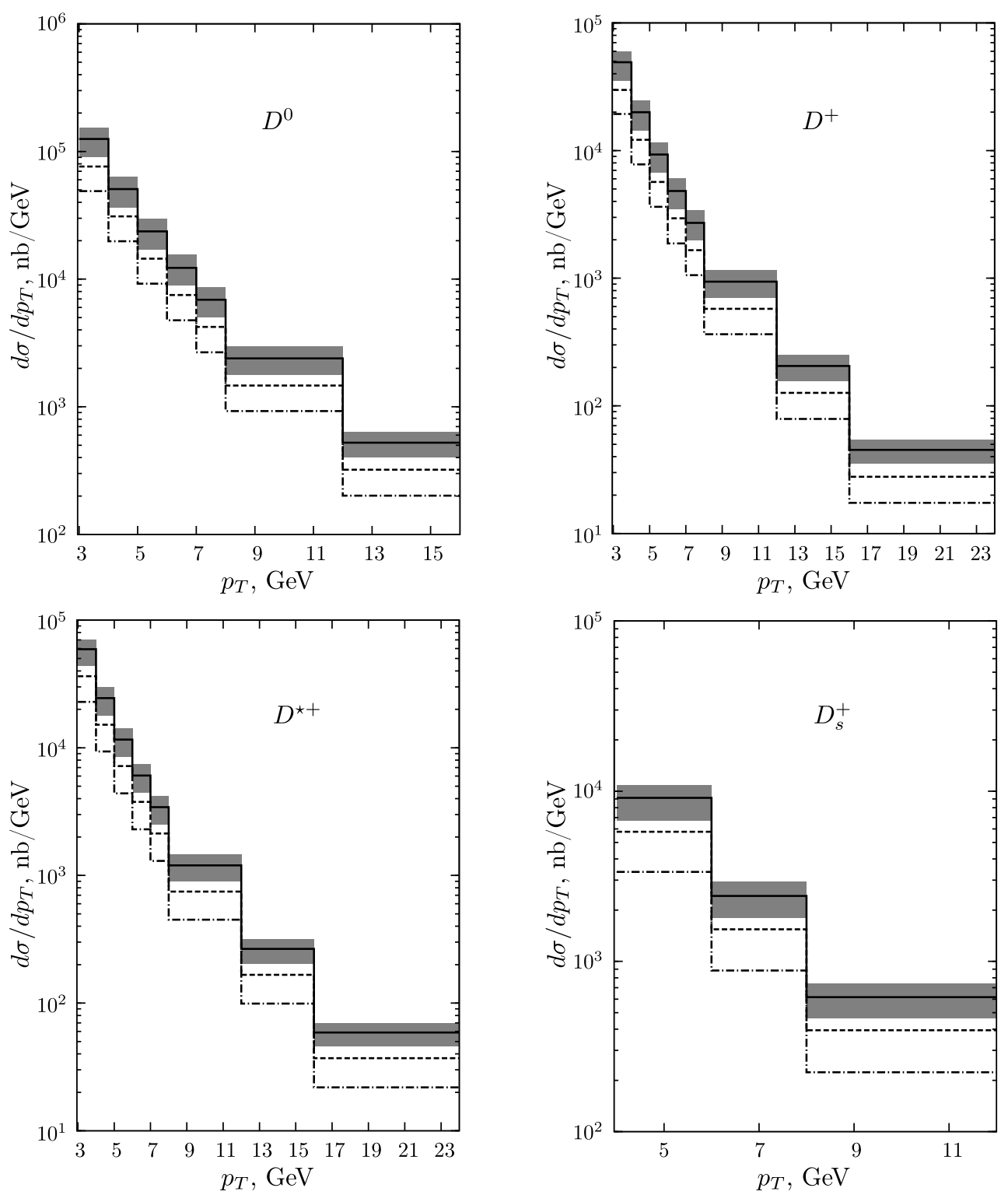

Рис. 5. Теоретические предсказания для распределений по поперечному импульсу для $D^{0}$ (вверху слева), $D^{+}$(вверху справа), $D^{\star+}$ (внизу слева), и $D_{s}^{+}$(внизу справа) мезонов в $p p$ рассеянии при $\sqrt{S}=14$ ТэВ и $|y|<0.5$, полученные в ЛП ТРП. Линии имеют те же обозначения, что и на рис. 3.

[Figure 5. Theoretical predictions for the transverse momentum distributions of $D^{0}$ (left, top), $D^{+}$(right, top), $D^{\star+}$ (left, bottom), and $D_{s}^{+}$(right, bottom) mesons in $p p$ scattering at $\sqrt{S}=14 \mathrm{TeV}$ and $|y|<0.5$ obtained in the LO PRA. The notations are as in Fig. 3] 
на порядок меньше, чем в случае рождения $D$-мезонов на Тэватроне. Наконец, на рис. 5 мы представили наши предсказания для планируемой энергии столкновений на БАК, равной $\sqrt{S}=14$ ТэВ, сохраняя другие кинематические параметры такими же, как и в работе [5].

Рассматривая рождение $D$-мезонов в центральной области быстрот, мы видим, что MPK подпроцесс (1) остаётся доминирующим при всех энергиях столкновений. В этом случае мы подтверждаем теоретическое предположение, упомянутое в предыдущем разделе, что МРК является лидирующим логарифмическим приближением в высокоэнергетических процессах рождения в БФКЛ подходе, в то время как КMPК оказывается следующим за лидирующим.

Заключение. В работе проведено исследование фрагментационного рождения $D^{0}, D^{+}, D^{\star+}$ и $D_{s}^{+}$мезонов в протон-(анти)протонных столкновениях в области центральных быстрот на Тэватроне и БАК в рамках теории реджезованных партонов. Мы использовали калибровочно инвариантные амплитуды жёстких партонных подпроцессов в ЛП теории с реджезованными глюонами в начальном состоянии вместе с неинтегрированными партонными ФР, предложенными Кимбером, Мартином и Рыскиным. Для описания непертурбативных переходов рождённых глюонов и $c$-кварков в $D$-мезоны мы применяли универсальные $\Phi \Phi$, полученные фитированием данных в $e^{+} e^{-}$ аннигиляции с коллайдера LEP1. Полученные нами результаты для рождения $D$-мезонов в центральной области быстрот находятся в хорошем согласии с экспериментальными данными БАК и хорошо согласуются с данными Тэватрона при больших поперечных импульсах.

Благодарности. Работа выполнена при финансовой поддержке Российского фонда фундаментальных исследований (проект № 14-02-00021-а) и гранта Президента РФ по государственной поддержке молодых российских ученых-кандидатов наук (грант MK-4150.2014.2).

\section{ORCIDs}

Антон Витальевич Карпишков: http://orcid.org/0000-0003-0762-5532

Максим Александрович Нефёдов: http://orcid.org//0000-0002-1046-9625

Владимир Анатольевич Салеев: http://orcid.org/0000-0003-0505-5564

Александра Викторовна Шипилова: http://orcid.org/0000-0003-3965-3757

\section{БИБЛИОГРАФИЧЕСКИЙ СПИСОК}

1. Карпишков А. В., Салеев В. А., Шипилова А. В. Рождение $D$-мезонов на Большом адронном коллайдере в реджевском пределе КХД/ Четвертая международная конферениия «Математическая физика и ее приложения»: материалы конф.; ред. чл.корр. РАН И. В. Волович; д.ф.-м.н., проф. В. П. Радченко. Самара: СамГТУ, 2014. C. 191-192.

2. Collins J. C., Ellis R. K. Heavy-quark production in very high energy hadron collisions // Nucl. Phys. B, 1991. vol. 360, no. 1. pp. 3-30. doi: 10.1016/0550-3213(91) 90288-9.

3. Gribov L. V., Levin E. M., Ryskin M. G. Semihard processes in QCD // Phys. Rep., 1983. vol. 100, no. 1-2. pp. 1-150. doi: 10.1016/0370-1573(83)90022-4.

4. Catani S., Ciafaloni K. M., Hautmann F. High energy factorization and small-x heavy flavour production// Nucl. Phys. B, 1991. vol.366, no.1. pp. 135-188. doi: 10.1016/ 0550-3213(91) 90055-3.

5. ALICE Collaboration (Abelev B., et al.) Measurement of charm production at central rapidity in proton-proton collisions at $\sqrt{s}=7 \mathrm{TeV} / /$ JHEP, 2012. vol. 2012, 128, arXiv: 1111.1553 [hep-ex]. doi : 10.1007/JHEP01(2012) 128.

6. ALICE Collaboration(Abelev B., et al.) $D_{s}^{+}$meson production at central rapidity in 
proton-proton collisions at $\sqrt{s}=7 \mathrm{TeV} / /$ Phys. Lett. B, 2012. vol.718, no. 2. pp. 279-294, arXiv: 1208.1948 [hep-ex]. doi: 10.1016/j.physletb.2012.10.049.

7. CDF II Collaboration (Acosta D., et al.) Measurement of Prompt Charm Meson Production Cross Sections in $p \bar{p}$ Collisions at $\sqrt{s}=1.96 \mathrm{TeV} / /$ Phys. Rev. Lett., 2003. vol. 91, no. 24, 241804, arXiv: hep-ex/0307080. doi : 10.1103/PhysRevLett.91.241804.

8. Kramer G., Spiesberger H. Inclusive $D^{*}$ production in photon-photon collisions at nextto-leading order QCD// Eur. Phys. J. C, 2001. vol.22, no. 2. pp. 289-301, arXiv: hepph/0109167. doi: 10.1007/s100520100805.

9. Kramer G., Spiesberger H. Inclusive $D$ production in $\gamma \gamma$ collisions: including the singleresolved contribution with massive quarks // Eur. Phys. J. C, 2003. vol. 28, no. 4. pp. 495513, arXiv: hep-ph/0302081. doi: 10.1140/epjc/s2003-01186-4.

10. Kramer G., Spiesberger H. Inclusive photoproduction of $D^{*}$ mesons with massive charm quarks // Eur. Phys. J. C, 2004. vol. 38, no. 3. pp. 309-318, arXiv: hep-ph/0311062. doi: 10. 1140/epjc/s2004-02054-5.

11. Kniehl B. A., Kramer G., Schienbein I., Spiesberger H. Inclusive $D^{* \pm}$ production in $p \bar{p}$ collisions with massive charm quarks // Phys. Rev. D, 2005. vol. 71, no. 1, 014018, arXiv: hep-ph/0410289. doi: 10.1103/physrevd.71.014018.

12. Kniehl B. A., Kramer G., Schienbein I., Spiesberger H. Collinear subtractions in hadroproduction of heavy quarks // Eur. Phys. J. C, 2005. vol. 41, no. 2. pp. 199-212, arXiv: hep-ph/0502194. doi : 10.1140/epjc/s2005-02200-7.

13. Cacciari M., Greco M., Nason P. The $p_{T}$ spectrum in heavy-flavour hadroproduction // J. High Energy Phys., 1998. vol.1998, no. 05, 007, arXiv: hep-ph/9803400. doi: 10.1088/ 1126-6708/1998/05/007.

14. Kniehl B. A., Kramer G. Charmed-hadron fragmentation functions from CERN LEP1 revisited // Phys. Rev. D, 2006. vol. 74, no. 3, 037502, arXiv: hep-ph/0607306. doi: 10.1103/ physrevd.74.037502.

15. Kniehl B. A., Kramer G., Schienbein I., Spiesberger H. Reconciling Open-Charm Production at the Fermilab Tevatron with QCD // Phys. Rev. Let., 2006. vol. 96, no. 1, 012001, arXiv: hep-ph/0508129. doi : 10.1103/physrevlett.96.012001.

16. Kniehl B. A., Kramer G., Schienbein I., Spiesberger H.// Eur. Phys. J. C, 2012. vol. 72, no. 7, 2082, arXiv: 1202.0439 [hep-ph]. doi: 10.1140/epjc/s10052-012-2082-2.

17. Грибов В. Н., Липатов Л. Н. Глубоко неупругое $e p$-рассеяние в теории возмущений // Ядерная физика, 1972. Т. 15, № 4. С. 781-807.

18. Докшицер Ю. Л. Вычисление структурных функций глубоко-неупругого рассеяния и $e^{+} e^{-}$-аннигиляции по теории возмущений в квантовой хромодинамике // ЖЭТФ, 1977. T. 73 , № 4. C. 1216-1240.

19. Altarelli G., Parisi G. Asymptotic freedom in parton language// Nucl. Phys. B, 1977. vol. 126, no. 2. pp. 298-318. doi : 10.1016/0550-3213(77) 90384-4.

20. OPAL Collaboration (Ackerstaff K., et al.) Measurement of $\mathrm{f}\left(\mathrm{c} \rightarrow \mathrm{D}^{*+} \mathrm{X}\right), \mathrm{f}\left(\mathrm{b} \rightarrow \mathrm{D}^{*+} \mathrm{X}\right)$ and $\Gamma_{\mathrm{c} \overline{\mathrm{c}}} / \Gamma_{\text {had }}$ using $\mathrm{D}^{* \pm}$ mesons // Eur. Phys. J. C, 1998. vol. 1, no. 3. pp. 439-459. doi: 10. $1007 / \mathrm{s} 100520050095$.

21. OPAL Collaboration(Alexander G., et al.) A study of charm hadron production in $Z^{0} \rightarrow c \bar{c}$ and $Z^{0} \rightarrow b \bar{b}$ decays at LEPdecays at LEP // Z. Phys. C - Particles and Fields, 1996. vol. 72, no. 1. pp. 1-16. doi: 10.1007/BF02909127.

22. Cacciari M., and Nason P. Charm cross sections for the Tevatron Run II // JHEP, 2003. vol. 2003, no. 09, 006, arXiv: hep-ph/0306212. doi : 10.1088/1126-6708/2003/09/006.

23. Cacciari M., Frixione S., Houdeau N., Mangano M. L., Nason P., Ridolfi G. Theoretical predictions for charm and bottom production at the LHC // JHEP, 2012. vol. 2012, no. 10, 137, arXiv: 1205.6344 [hep-ph]. doi: 10.1007/jhep10(2012) 137.

24. Maciuła R., Szczurek A. Open charm production at the LHC: $k_{t}$-factorization approach // Phys. Rev. D, 2013. vol.87, no. 9, 094022, arXiv: 1301.3033 [hep-ph]. doi: 10.1103/ physrevd.87.094022. 
25. Kniehl B. A., Saleev V. A., Shipilova A. V., Yatsenko E. V. Single jet and prompt-photon inclusive production with multi-Regge kinematics: From Tevatron to LHC // Phys. Rev. D, 2011. vol. 84, no. 7, 074017, arXiv: 1107.1462 [hep-ph]. doi : 10.1103/physrevd.84.074017.

26. Nefedov M. A., Saleev V. A., Shipilova A. V. Dijet azimuthal decorrelations at the LHC in the parton Reggeization approach // Phys. Rev. D, 2013. vol.87, no. 9, 094030, arXiv: 1304.3549 [hep-ph]. doi: 10.1103/physrevd.87.094030.

27. Saleev V. A. Deep inelastic scattering and prompt photon production within the framework of the quark Reggeization hypothesis // Phys. Rev. D, 2008. vol.78, no. 3, 034033, arXiv: 0807.1587 [hep-ph]. doi : 10.1103/physrevd.78.034033.

28. Saleev V. A. Prompt photon photoproduction at HERA within the framework of the quark Reggeization hypothesis // Phys. Rev. D, 2008. vol. 78, no. 11, 114031, arXiv: 0812.0946 [hepph]. doi: 10.1103/physrevd.78.114031.

29. Kniehl B. A., Nefedov M. A., Saleev V. A. Prompt-photon plus jet associated photoproduction at HERA in the parton Reggeization approach // Phys. Rev. D, 2014. vol. 89, no. 11, 114016, arXiv: 1404.3513 [hep-ph]. doi: 10.1103/physrevd.89.114016.

30. Nefedov M. A., Nikolaev N. N., Saleev V. A. Drell-Yan lepton pair production at high energies in the parton Reggeization approach // Phys. Rev. D, 2013. vol. 87, no. 1, 014022, arXiv: 1211.5539 [hep-ph]. doi: 10.1103/physrevd.87.014022.

31. Kniehl B. A., Saleev V. A., Shipilova A. V. Inclusive $b$ and $b \bar{b}$ production with quasimulti-Regge kinematics at the Tevatron // Phys. Rev. D, 2010. vol. 81, no. 9, 094010, arXiv: 1003.0346 [hep-ph]. doi: 10.1103/physrevd.81.094010.

32. Saleev V. A., Shipilova A. V. Inclusive $b$-jet and $b \bar{b}$-dijet production at the LHC via Reggeized gluons // Phys. Rev. D, 2012. vol. 86, no. 3, arXiv: 1201.4640 [hep-ph]. doi: 10. 1103/physrevd.86.034032.

33. Kniehl B. A., Vasin D. V., Saleev V. A. Charmonium production at high energy in the $k_{T^{-}}$ factorization approach // Phys. Rev. D, 2006. vol. 73, no. 7, 074022, arXiv: hep-ph/0602179. doi: 10.1103/physrevd.73.074022.

34. Kniehl B. A., Saleev V. A., Vasin D. V. Bottomonium production in the Regge limit of QCD // Phys. Rev. D, 2006. vol.74, no. 1, 014024, arXiv: hep-ph/0607254. doi: 10.1103/ physrevd.74.014024.

35. Saleev V. A., Vasin D. V. Direct $J / \psi$ and $\psi^{\prime}$ hadroproduction via fragmentation in the collinear parton model and $k_{T}$-factorization approach // Phys. Rev. D, 2003. vol.68, no. 11, 114013, arXiv: hep-ph/0304114. doi: 10.1103/physrevd.68.114013.

36. Салеев В. А., Васин Д. В. Адронное рождение прямых $J / \psi$ - и $\psi^{\prime}$-мезонов в процессах фрагментации глюонов и с-кварков при высоких энергиях // Ядерная физика, 2005. T. 68 , № 1. C. 95-105.

37. Saleev V. A., Nefedov M. A., Shipilova A. V. Prompt $J / \psi$ production in the Regge limit of QCD: From the Tevatron to the LHC // Phys. Rev. D, 2012. vol.85, no. 7, 074013, arXiv: 1201.3464 [hep-ph]. doi : 10.1103/physrevd.85.074013.

38. Nefedov M. A., Saleev V. A., Shipilova A. V. Prompt $\Upsilon(n S)$ production at the LHC in the Regge limit of QCD // Phys. Rev. D, 2013. vol. 88, no. 1, 014003, arXiv: 1305.7310 [hep-ph]. doi: 10.1103/physrevd.88.014003.

39. Кураев Э. А., Липатов Л. Н., Фадин В. С. Мультиреджевские процессы в теории Янга-Миллса // ЖЭТФ, 1976. Т. 71, № 3. С. 840-855.

40. Балицкий Я. Я., Липатов Л. Н. Сингулярность Померанчука в квантовой хромодинамике // Ядерная физика, 1978. Т. 28. С. 1597-1611.

41. Fadin V. S., Kuraev E. A., Lipatov L. N. On the Pomeranchuk singularity in asymptotically free theories // Phys. Lett. B, 1975. vol.60, no.1. pp. 50-52. doi:10.1016/0370-2693(75) 90524-9.

42. Fadin V. S., Fiore R. Calculation of Reggeon vertices in QCD // Phys. Rev. D, 2001. vol. 64, no. 11, 114012, arXiv: hep-ph/0107010. doi: 10.1103/physrevd.64.114012.

43. Lipatov L. N. Gauge invariant effective action for high energy processes in QCD // Nucl. Phys. B, 1995. vol.452, no.1-2. pp. 369-397, arXiv: hep-ph/9502308. doi: 10.1016/ 0550-3213 (95) 00390-e. 
44. Lipatov L. N., Vyazovsky M. I. Quasi-multi-Regge processes with a quark exchange in the $t$-channel // Nucl. Phys. B, 2001. vol. 597, no. 1-3. pp. 399-409, arXiv: hep-ph/0009340. doi: 10.1016/s0550-3213(00)00709-4.

45. Antonov E. N., Cherednikov I. O., Kuraev E. A., Lipatov L. N. Feynman rules for effective Regge action // Nucl. Phys. B, 2005. vol. 721, no. 1-3. pp. 111-135, arXiv: hep-ph/0411185. doi: 10.1016/j.nuclphysb.2005.05.013.

46. Kimber M. A., Martin A. D., Ryskin M. G. Unintegrated parton distributions // Phys. Rev. D, 2001. vol.63, no. 11, 114027, arXiv: hep-ph/0101348. doi: 10.1103/physrevd.63. 114027.

47. Ciafaloni M. Coherence effects in initial jets at small $Q^{2} /$ s // Nucl. Phys. B, 1988. vol. 296, no. 1. pp. 49-74. doi : 10.1016/0550-3213(88)90380-x.

48. Catani S., Fiorani F., Marchesini G. QCD coherence in initial state radiation // Phys. Lett. B, 1990. vol. 234, no. 3. pp. 339-345. doi : 10.1016/0370-2693(90) 91938-8.

49. Catani S., Fiorani F., Marchesini G. Small-x behaviour of initial state radiation in perturbative QCD// Nucl. Phys. B, 1990. vol.336, no.1. pp. 18-85. doi:10.1016/ 0550-3213 (90) 90342-b.

50. CDF Collaboration (Abe F., et al.) $J / \psi$ and $\psi(2 S)$ Production in $p \bar{p}$ Collisions at $\sqrt{s}=1.8 \mathrm{TeV} / /$ Phys. Rev. Lett., 1997. vol.79, no.4, 572. doi: http://dx.doi.org/10. 1103/PhysRevLett.79.572.

51. CDF Collaboration (Abe F., et al.) Production of $J / \psi$ Mesons from $\chi_{c}$ Meson Decays in $p \bar{p}$ Collisions at $\sqrt{s}=1.8 \mathrm{TeV} / /$ Phys. Rev. Lett., 1997. vol. 79, no. 4, 578. doi: 10.1103/ PhysRevLett.79.578.

52. CDF Collaboration (Affolder T., et al.) Measurement of $J / \psi$ and $\psi(2 S)$ Polarization in $p \bar{p}$ Collisions at $\sqrt{s}=1.8 \mathrm{TeV} / /$ Phys. Rev. Lett., 2000. vol. 85, no. 14, 2886. doi: 10.1103/ PhysRevLett. 85.2886.

53. CDF Collaboration (Acosta D., et al.) Measurement of the $J / \psi$ meson and $b$-hadron production cross sections in $p \bar{p}$ collisions at $\sqrt{s}=1960 \mathrm{GeV} / /$ Phys. Rev. D, 2005. vol. 71, no. 3, 032001, arXiv: hep-ex/0412071. doi : 10.1103/PhysRevD.71.032001.

54. CDF Collaboration (Abe F., et al.) $\Upsilon$ Production in $p \bar{p}$ Collisions at $\sqrt{s}=1.8 \mathrm{TeV} / /$ Phys. Rev. Lett., 1995. vol. 75, no. 24, 4358. doi: 10.1103/PhysRevLett.75.4358.

55. CDF Collaboration (Acosta D., et al.) $\Upsilon$ Production and Polarization in $p \bar{p}$ Collisions at $\sqrt{s}=1.8 \mathrm{TeV} / /$ Phys. Rev. Lett., 2002. vol. 88, no. 16, 161802. doi: 10.1103/PhysRevLett. 88.161802 .

56. C0 Collaboration (Abazov V. M., et al.) Measurement of Inclusive Differential Cross Sections for $\Upsilon(1 S)$ Production in $p \bar{p}$ Collisions at $\sqrt{s}=1.96 \mathrm{TeV} / /$ Phys. Rev. Lett., 2005. vol. 94, no. 23, 232001, arXiv: hep-ex/0502030. doi: 10.1103/PhysRevLett.94.232001 ; Erratum // Phys. Rev. Lett., 2008. vol. 100, no. 4, 049902. doi: 10.1103/PhysRevLett.100.049902.

57. ATLAS Collaboration (Aad G., et al.) Measurement of upsilon production in $7 \mathrm{TeV}$ $p p$ collisions at ATLAS // Phys. Rev. D, 2013. vol. 87, 052004, arXiv: 1211.7255 [hep-ex]. doi: 10.1103/PhysRevD.87.052004.

58. CMS Collaboration (Khachatryan V., et al.) Upsilon production cross section in pp collisions at $\sqrt{s}=7 \mathrm{TeV} / /$ Phys. Rev. D, 2011. vol. 83, 112004. doi: 10.1103/PhysRevD.83.112004.

59. LHCb Collaboration (Aaij R., et al.) Measurement of $\Upsilon$ production in $p p$ collisions at $\sqrt{s}=7 \mathrm{TeV} / /$ Eur. Phys. J. C, 2012. vol.72, 2025, arXiv: 1202.6579 [hep-ex]. doi: 10 . 1140/epjc/s10052-012-2025-y.

60. Mele B., Nason P. The fragmentation function for heavy quarks in QCD // Nucl. Phys B, 1991. vol. 361, no. 3. pp. 626-644. doi: 10.1016/0550-3213(91)90597-q.

61. Fadin V. S., Lipatov L. N. Next-to-leading corrections to the BFKL equation from gluon and quark production // Nucl. Phys. B, 1996. vol. 477, no. 3. pp. 767-805, arXiv: hep-ph/9602287. doi : 10.1016/0550-3213(96)00334-3.

Поступила в редакцию 24/I/2015; в окончательном варианте - $17 /$ III $/ 2015$; принята в печать - 08/IV/2015. 
Vestn. Samar. Gos. Techn. Un-ta. Ser. Fiz.-mat. nauki

[J. Samara State Tech. Univ., Ser. Phys. \& Math. Sci.], 2015, vol. 19, no. 3, pp. 441-461

ISSN: 2310-7081 (online), 1991-8615 (print)

doi: http://dx.doi.org/10.14498/vsgtu1402

MSC: 81T13, 81U35

\title{
$D$-MESON PRODUCTION AT LARGE HADRON COLLIDER IN THE REGGE LIMIT OF QCD*
}

\author{
A. V. Karpishkov, M. A. Nefedov, \\ V. A. Saleev, A. V. Shipilova
}

Samara State University,

1, Academician Pavlov st., Samara, 443011, Russian Federation.

\begin{abstract}
We study the inclusive production of $D^{0}, D^{+}, D^{\star+}$, and $D_{s}^{+}$mesons in proton-antiproton collisions at the Tevatron and in proton-proton collisions at LHC at leading order in the parton Reggeization approach endowed with universal fragmentation functions fitted to $e^{+} e^{-}$annihilation data from CERN LEP1. We have described $D$-meson transverse momentum distributions measured in the central region of rapidity by the CDF Collaboration at Tevatron $(|y|<1)$ and ALICE Collaboration at LHC $(|y|<0.5)$ within uncertainties and without free parameters, using Kimber-MartinRyskin unintegrated gluon distribution function in a proton. The $2 \rightarrow 1$ hard subprocess of gluon production via a fusion of two Reggeized gluons in the PRA framework is proposed for the first time in the case of $D$-meson fragmentation production and proved to be a dominant one. We found our results for $D$-meson central-rapidity production are in the good agreement with experimental data from the LHC and with large-transverse-momenta Tevatron data. The achieved degree of agreement for the LHC exceeds the one obtained by NLO calculations in the conventional collinear parton model and previous LO calculations in $k_{T}$-factorization without taking into account the $2 \rightarrow 1$ subprocess. The predictions for the $D$-meson production in the central rapidity region for the expected LHC energy of $\sqrt{S}=14 \mathrm{TeV}$ are also presented.
\end{abstract}

(C) 2015 Samara State Technical University.

Please cite this article in press as:

Karpishkov A. V., Nefedov M. A., Saleev V. A., Shipilova A. V. D-meson production at Large Hadron Collider in the Regge limit of QCD, Vestn. Samar. Gos. Tekhn. Univ., Ser. Fiz.-Mat. Nauki [J. Samara State Tech. Univ., Ser. Phys. \& Math. Sci.], 2015, vol. 19, no. 3, pp. 441-461. doi: 10.14498/vsgtu1402. (In Russian)

\section{Authors Details:}

Anton V. Karpishkov (karpishkov@rambler.ru), Postgraduate Student, Dept. of General \& Theoretical Physics.

Maxim A. Nefedov (nefedovma@gmail.com), Postgraduate Student, Dept. of General \& Theoretical Physics.

Vladimir A. Saleev (Dr. Phys. \& Math. Sci.; saleev@samsu.ru; Corresponding Author), Professor, Dept. of General \& Theoretical Physics.

Alexandra A. Shipilova (Cand. Phys. \& Math. Sci.; alexshipilova@samsu.ru), Scientific Researcher, Lab. of Mathematical Modeling of Materials, the Samara Center of Theoretical Materials Science, SSU.

${ }^{*}$ This paper is an extended version of the paper [1], presented at the Mathematical Physics and Its Applications 2014 Conference. 
Keywords: quantum chromodynamics, $k_{T}$-factorization, parton reggeization approach, high energy physics, charmed mesons.

doi: http://dx.doi.org/10.14498/vsgtu1402

Acknowledgments. This work was supported by the Russian Foundation for Basic Research (Project No. 14-02-00021-a) and by the Grant of President of Russian Federation (Grant No. MK-4150.2014.2).

\section{ORCIDs}

Anton V. Karpishkov: http://orcid.org/0000-0003-0762-5532

Maxim A. Nefedov: http://orcid.org//0000-0002-1046-9625

Vladimir A. Saleev: http://orcid.org/0000-0003-0505-5564

Alexandra A. Shipilova: http://orcid.org/0000-0003-3965-3757

\section{REFERENCES}

1. Karpishkov A. V., Saleev V. A., Shipilova A. V. D-meson production at Large Hadron Collider in the Regge limit of $\mathrm{QCD}$, The 4nd International Conference "Mathematical Physics and its Applications", Book of Abstracts and Conference Materials; eds. I. V. Volovich; V. P. Radchenko. Samara, Samara State Technical Univ., 2014, pp. 191192 (In Russian).

2. Collins J. C., Ellis R. K. Heavy-quark production in very high energy hadron collisions, Nucl. Phys. B, 1991, vol. 360, no. 1, pp. 3-30. doi : 10.1016/0550-3213(91)90288-9.

3. Gribov L. V., Levin E. M., Ryskin M. G. Semihard processes in QCD, Phys. Rep., 1983, vol. 100, no. 1-2, pp. 1-150. doi: 10.1016/0370-1573(83)90022-4.

4. Catani S., Ciafaloni K. M., Hautmann F. High energy factorization and small-x heavy flavour production, Nucl. Phys. B, 1991, vol.366, no. 1, pp. 135-188. doi: 10.1016/0550-3213(91) 90055-3.

5. ALICE Collaboration (Abelev B., et al.) Measurement of charm production at central rapidity in proton-proton collisions at $\sqrt{s}=7 \mathrm{TeV}$, JHEP, 2012, vol. 2012, 128, arXiv: 1111.1553 [hep-ex]. doi : 10.1007/JHEP01 (2012) 128.

6. ALICE Collaboration(Abelev B., et al.) $D_{s}^{+}$meson production at central rapidity in proton-proton collisions at $\sqrt{s}=7 \mathrm{TeV}$, Phys. Lett. B, 2012, vol.718, no. 2, pp. 279-294, arXiv: 1208.1948 [hep-ex]. doi: 10.1016/j.physletb.2012.10.049.

7. CDF II Collaboration (Acosta D., et al.) Measurement of Prompt Charm Meson Production Cross Sections in $p \bar{p}$ Collisions at $\sqrt{s}=1.96 \mathrm{TeV}$, Phys. Rev. Lett., 2003, vol.91, no. 24, 241804, arXiv: hep-ex/0307080. doi: 10.1103/PhysRevLett.91.241804.

8. Kramer G., Spiesberger H. Inclusive $D^{*}$ production in photon-photon collisions at nextto-leading order QCD, Eur. Phys. J. C, 2001, vol.22, no. 2, pp. 289-301, arXiv: hepph/0109167. doi: 10.1007/s100520100805.

9. Kramer G., Spiesberger H. Inclusive $D$ production in $\gamma \gamma$ collisions: including the singleresolved contribution with massive quarks, Eur. Phys. J. C, 2003, vol. 28, no. 4, pp. 495-513, arXiv: hep-ph/0302081. doi: 10.1140/epjc/s2003-01186-4.

10. Kramer G., Spiesberger H. Inclusive photoproduction of $D^{*}$ mesons with massive charm quarks, Eur. Phys. J. C, 2004, vol.38, no. 3, pp. 309-318, arXiv: hep-ph/0311062. doi: 10. 1140/epjc/s2004-02054-5.

11. Kniehl B. A., Kramer G., Schienbein I., Spiesberger H. Inclusive $D^{* \pm}$ production in $p \bar{p}$ collisions with massive charm quarks, Phys. Rev. D, 2005, vol.71, no. 1, 014018, arXiv: hep-ph/0410289. doi: 10.1103/physrevd.71.014018.

12. Kniehl B. A., Kramer G., Schienbein I., Spiesberger H. Collinear subtractions in hadroproduction of heavy quarks, Eur. Phys. J. C, 2005, vol. 41, no. 2, pp. 199-212, arXiv: hep-ph/0502194. doi: 10.1140/epjc/s2005-02200-7.

13. Cacciari M., Greco M., Nason P. The $p_{T}$ spectrum in heavy-flavour hadroproduction, J. High Energy Phys., 1998, vol.1998, no. 05, 007, arXiv: hep-ph/9803400. doi: 10.1088/ 1126-6708/1998/05/007. 
14. Kniehl B. A., Kramer G. Charmed-hadron fragmentation functions from CERN LEP1 revisited, Phys. Rev. D, 2006, vol.74, no. 3, 037502, arXiv: hep-ph/0607306. doi: 10.1103/ physrevd.74.037502.

15. Kniehl B. A., Kramer G., Schienbein I., Spiesberger H. Reconciling Open-Charm Production at the Fermilab Tevatron with QCD, Phys. Rev. Let., 2006, vol.96, no. 1, 012001, arXiv: hep-ph/0508129. doi: 10.1103/physrevlett.96.012001.

16. Kniehl B. A., Kramer G., Schienbein I., Spiesberger H., Eur. Phys. J. C, 2012, vol. 72, no. 7, 2082, arXiv: 1202.0439 [hep-ph]. doi: 10.1140/epjc/s10052-012-2082-2.

17. Gribov V. N., Lipatov L. N. Deep inelastic ep scattering in perturbation theory, Sov. J. Nucl. Phys., 1972, vol. 15, no. 4, pp. 438-450.

18. Dokshitser Yu. L. Calculation of structure functions of deep-inelastic scattering and $e^{+} e^{-}$ annihilation by perturbation theory in quantum chromodynamics, Sov. Phys. JETP, 1977, vol. 46, no. 4, pp. 641-653, http://www.jetp.ac.ru/cgi-bin/dn/e_046_04_0641.pdf.

19. Altarelli G., Parisi G. Asymptotic freedom in parton language, Nucl. Phys. B, 1977, vol. 126, no. 2, pp. 298-318. doi: 10.1016/0550-3213(77) 90384-4.

20. OPAL Collaboration (Ackerstaff K., et al.) Measurement of $\mathrm{f}\left(\mathrm{c} \rightarrow \mathrm{D}^{*+} \mathrm{X}\right), \mathrm{f}\left(\mathrm{b} \rightarrow \mathrm{D}^{*+} \mathrm{X}\right)$ and $\Gamma_{\mathrm{c} \bar{c}} / \Gamma_{\text {had }}$ using $\mathrm{D}^{* \pm}$ mesons, Eur. Phys. J. C, 1998, vol. 1, no. 3, pp. 439-459. doi: 10.1007/ s100520050095.

21. OPAL Collaboration(Alexander G., et al.) A study of charm hadron production in $Z^{0} \rightarrow c \bar{c}$ and $Z^{0} \rightarrow b \bar{b}$ decays at LEPdecays at LEP, Z. Phys. $C$ - Particles and Fields, 1996, vol. 72 , no. 1, pp. 1-16. doi : 10.1007/BF02909127.

22. Cacciari M., and Nason P. Charm cross sections for the Tevatron Run II, JHEP, 2003, vol. 2003, no. 09, 006, arXiv: hep-ph/0306212. doi: 10.1088/1126-6708/2003/09/006.

23. Cacciari M., Frixione S., Houdeau N., Mangano M. L., Nason P., Ridolfi G. Theoretical predictions for charm and bottom production at the LHC, JHEP, 2012, vol. 2012, no. 10, 137, arXiv: 1205.6344 [hep-ph]. doi : 10.1007/jhep10(2012) 137.

24. Maciuła R., Szczurek A. Open charm production at the LHC: $k_{t}$-factorization approach, Phys. Rev. D, 2013, vol.87, no. 9, 094022, arXiv: 1301.3033 [hep-ph]. doi: 10.1103/ physrevd.87.094022.

25. Kniehl B. A., Saleev V. A., Shipilova A. V., Yatsenko E. V. Single jet and prompt-photon inclusive production with multi-Regge kinematics: From Tevatron to LHC, Phys. Rev. D, 2011, vol. 84, no. 7, 074017, arXiv: 1107.1462 [hep-ph]. doi: 10.1103/physrevd.84.074017.

26. Nefedov M. A., Saleev V. A., Shipilova A. V. Dijet azimuthal decorrelations at the LHC in the parton Reggeization approach, Phys. Rev. D, 2013, vol.87, no. 9, 094030, arXiv: 1304.3549 [hep-ph]. doi: 10.1103/physrevd.87.094030.

27. Saleev V. A. Deep inelastic scattering and prompt photon production within the framework of the quark Reggeization hypothesis, Phys. Rev. D, 2008, vol.78, no. 3, 034033, arXiv: 0807.1587 [hep-ph]. doi : 10.1103/physrevd.78.034033.

28. Saleev V. A. Prompt photon photoproduction at HERA within the framework of the quark Reggeization hypothesis, Phys. Rev. D, 2008, vol. 78, no. 11, 114031, arXiv: 0812.0946 [hepph]. doi: 10.1103/physrevd.78.114031.

29. Kniehl B. A., Nefedov M. A., Saleev V. A. Prompt-photon plus jet associated photoproduction at HERA in the parton Reggeization approach, Phys. Rev. D, 2014, vol. 89, no. 11, 114016, arXiv: 1404.3513 [hep-ph]. doi: 10.1103/physrevd.89.114016.

30. Nefedov M. A., Nikolaev N. N., Saleev V. A. Drell-Yan lepton pair production at high energies in the parton Reggeization approach, Phys. Rev. D, 2013, vol.87, no. 1, 014022, arXiv: 1211.5539 [hep-ph]. doi: 10.1103/physrevd.87.014022.

31. Kniehl B. A., Saleev V. A., Shipilova A. V. Inclusive $b$ and $b \bar{b}$ production with quasimulti-Regge kinematics at the Tevatron, Phys. Rev. D, 2010, vol.81, no. 9, 094010, arXiv: 1003.0346 [hep-ph]. doi : 10.1103/physrevd.81.094010.

32. Saleev V. A., Shipilova A. V. Inclusive $b$-jet and $b \bar{b}$-dijet production at the LHC via Reggeized gluons, Phys. Rev. D, 2012, vol. 86, no. 3, arXiv: 1201.4640 [hep-ph]. doi: 10 . 1103/physrevd.86.034032. 
33. Kniehl B. A., Vasin D. V., Saleev V. A. Charmonium production at high energy in the $k_{T^{-}}$ factorization approach, Phys. Rev. D, 2006, vol.73, no. 7, 074022, arXiv: hep-ph/0602179. doi: 10.1103/physrevd.73.074022.

34. Kniehl B. A., Saleev V. A., Vasin D. V. Bottomonium production in the Regge limit of QCD, Phys. Rev. D, 2006, vol.74, no. 1, 014024, arXiv: hep-ph/0607254. doi: 10.1103/ physrevd.74.014024.

35. Saleev V. A., Vasin D. V. Direct $J / \psi$ and $\psi^{\prime}$ hadroproduction via fragmentation in the collinear parton model and $k_{T}$-factorization approach, Phys. Rev. D, 2003, vol.68, no. 11, 114013, arXiv: hep-ph/0304114. doi: 10.1103/physrevd.68.114013.

36. Saleev V. A., Vasin D. V. Hadroproduction of direct $J / \psi$ and $\psi^{\prime}$ mesons in the fragmentation of gluons and c quarks at high energies, Phys. Atom. Nucl., 2005, vol. 68, no. 1, pp. 94-103. doi: $10.1134 / 1.1858562$.

37. Saleev V. A., Nefedov M. A., Shipilova A. V. Prompt $J / \psi$ production in the Regge limit of QCD: From the Tevatron to the LHC, Phys. Rev. D, 2012, vol. 85, no. 7, 074013, arXiv: 1201.3464 [hep-ph]. doi: 10.1103/physrevd.85.074013.

38. Nefedov M. A., Saleev V. A., Shipilova A. V. Prompt $\Upsilon(n S)$ production at the LHC in the Regge limit of QCD, Phys. Rev. D, 2013, vol. 88, no. 1, 014003, arXiv: 1305.7310 [hep-ph]. doi: 10.1103/physrevd.88.014003.

39. Kuraev E. A., Lipatov L. N., Fadin V. S. Multiregge processes in the Yang-Mills theory, Sov. Phys. JETP, 1976, vol.44, no.3, pp. 443-451, http://www.jetp.ac.ru/cgi-bin/dn/ e_044_03_0443.pdf.

40. Balitsky I. I., Lipatov L. N. The Pomeranchuk Singularity, Process, Theory, Sov. J. Nucl. Phys., 1978, vol. 28, pp. 822-829.

41. Fadin V. S., Kuraev E. A., Lipatov L. N. On the Pomeranchuk singularity in asymptotically free theories, Phys. Lett. B, 1975, vol.60, no. 1, pp. 50-52. doi:10.1016/0370-2693(75) 90524-9.

42. Fadin V. S., Fiore R. Calculation of Reggeon vertices in QCD, Phys. Rev. D, 2001, vol. 64, no. 11, 114012, arXiv: hep-ph/0107010. doi: 10.1103/physrevd.64.114012.

43. Lipatov L. N. Gauge invariant effective action for high energy processes in QCD, Nucl. Phys. B, 1995, vol. 452, no.1-2, pp. 369-397, arXiv: hep-ph/9502308. doi: 10.1016/ 0550-3213(95) 00390-e.

44. Lipatov L. N., Vyazovsky M. I. Quasi-multi-Regge processes with a quark exchange in the $t$-channel, Nucl. Phys. B, 2001, vol.597, no. 1-3, pp. 399-409, arXiv: hep-ph/0009340. doi: 10.1016/s0550-3213(00)00709-4.

45. Antonov E. N., Cherednikov I. O., Kuraev E. A., Lipatov L. N. Feynman rules for effective Regge action, Nucl. Phys. B, 2005, vol.721, no.1-3, pp. 111-135, arXiv: hep-ph/0411185. doi: 10.1016/j.nuclphysb.2005.05.013.

46. Kimber M. A., Martin A. D., Ryskin M. G. Unintegrated parton distributions, Phys. Rev. D, 2001, vol. 63, no. 11, 114027, arXiv: hep-ph/0101348. doi: 10.1103/physrevd.63.114027.

47. Ciafaloni M. Coherence effects in initial jets at small $Q^{2} / s$, Nucl. Phys. B, 1988, vol. 296, no. 1, pp. 49-74. doi : 10.1016/0550-3213(88)90380-x.

48. Catani S., Fiorani F., Marchesini G. QCD coherence in initial state radiation, Phys. Lett. B, 1990, vol. 234, no. 3, pp. 339-345. doi: 10.1016/0370-2693(90)91938-8.

49. Catani S., Fiorani F., Marchesini G. Small-x behaviour of initial state radiation in perturbative QCD, Nucl. Phys. B, 1990, vol.336, no.1, pp. 18-85. doi:10.1016/ 0550-3213 (90) 90342-b.

50. CDF Collaboration (Abe F., et al.) $J / \psi$ and $\psi(2 S)$ Production in $p \bar{p}$ Collisions at $\sqrt{s}=1.8 \mathrm{TeV}$, Phys. Rev. Lett., 1997, vol.79, no. 4, 572. doi: http://dx.doi.org/10.1103/ PhysRevLett.79.572.

51. CDF Collaboration (Abe F., et al.) Production of $J / \psi$ Mesons from $\chi_{c}$ Meson Decays in $p \bar{p}$ Collisions at $\sqrt{s}=1.8 \mathrm{TeV}$, Phys. Rev. Lett., 1997, vol.79, no. 4, 578. doi: 10.1103/ PhysRevLett. 79.578. 
52. CDF Collaboration (Affolder T., et al.) Measurement of $J / \psi$ and $\psi(2 S)$ Polarization in $p \bar{p}$ Collisions at $\sqrt{s}=1.8 \mathrm{TeV}$, Phys. Rev. Lett., 2000, vol. 85, no. 14, 2886. doi: 10.1103/ PhysRevLett.85.2886.

53. CDF Collaboration (Acosta D., et al.) Measurement of the $J / \psi$ meson and $b$-hadron production cross sections in $p \bar{p}$ collisions at $\sqrt{s}=1960 \mathrm{GeV}$, Phys. Rev. D, 2005, vol. 71, no. 3, 032001, arXiv: hep-ex/0412071. doi: 10.1103/PhysRevD.71.032001.

54. CDF Collaboration (Abe F., et al.) $\Upsilon$ Production in $p \bar{p}$ Collisions at $\sqrt{s}=1.8 \mathrm{TeV}$, Phys. Rev. Lett., 1995, vol. 75, no. 24, 4358. doi: 10.1103/PhysRevLett.75.4358.

55. CDF Collaboration (Acosta D., et al.) $\Upsilon$ Production and Polarization in $p \bar{p}$ Collisions at $\sqrt{s}=1.8 \mathrm{TeV}$, Phys. Rev. Lett., 2002, vol.88, no. 16, 161802. doi: 10.1103/PhysRevLett. 88.161802 .

56. C0 Collaboration (Abazov V. M., et al.) Measurement of Inclusive Differential Cross Sections for $\Upsilon(1 S)$ Production in $p \bar{p}$ Collisions at $\sqrt{s}=1.96 \mathrm{TeV}$, Phys. Rev. Lett., 2005, vol.94, no. 23, 232001, arXiv: hep-ex/0502030. doi: 10.1103/PhysRevLett.94.232001 ; Erratum, Phys. Rev. Lett., 2008, vol. 100, no. 4, 049902. doi: 10.1103/PhysRevLett.100.049902.

57. ATLAS Collaboration (Aad G., et al.) Measurement of upsilon production in $7 \mathrm{TeV} p p$ collisions at ATLAS, Phys. Rev. D, 2013, vol. 87, 052004, arXiv: 1211.7255 [hep-ex]. doi : 10. 1103/PhysRevD . 87.052004.

58. CMS Collaboration (Khachatryan V., et al.) Upsilon production cross section in pp collisions at $\sqrt{s}=7 \mathrm{TeV}$, Phys. Rev. D, 2011, vol.83, 112004. doi: 10.1103/PhysRevD.83.112004.

59. LHCb Collaboration (Aaij R., et al.) Measurement of $\Upsilon$ production in $p p$ collisions at $\sqrt{s}=7 \mathrm{TeV}$, Eur. Phys. J. C, 2012, vol. 72, 2025, arXiv: 1202.6579 [hep-ex]. doi: 10.1140/ epjc/s10052-012-2025-y.

60. Mele B., Nason P. The fragmentation function for heavy quarks in QCD, Nucl. Phys B, 1991, vol. 361, no. 3, pp. 626-644. doi: 10.1016/0550-3213(91)90597-q.

61. Fadin V. S., Lipatov L. N. Next-to-leading corrections to the BFKL equation from gluon and quark production, Nucl. Phys. B, 1996, vol. 477, no. 3, pp. 767-805, arXiv: hep-ph/9602287. doi : 10.1016/0550-3213(96)00334-3.

Received 24/I/2015;

received in revised form $17 / \mathrm{III} / 2015$;

accepted 08/IV/2015. 